\title{
Briefing: Food waste - next steps for food processors and manufacturers
}

\author{
Jeff Cooper BSc, MSc, FRGS, FCIWM \\ Independent Consultant on Renewable Energy from Waste and \\ Technical Editor, Jeff Cooper Environment, London, UK
}

(jeffcooper.101@btinternet.com)

The Courtauld Commitment 2025 sets ambitious targets for food waste prevention and reduction for the UK, enveloped within the overall target for reducing the environmental impacts of food waste by $20 \%$ by 2025 compared with that of 2015. In addition, there is the UN Sustainable Development Goal $12 \cdot 3$ to reduce global food waste by $\mathbf{5 0} \%$ by $\mathbf{2 0 3 0}$. This is an objective adopted by a number of businesses and governments, albeit there will, as always, be problems with the calculation of the measurement protocol.

\section{Introduction}

This briefing article focuses on the following aspects of the programme, which have implications for businesses throughout the whole of the food supply chain.

- The continuation of work of the Hospitality and Food Service Agreement (HaFSA), which had finished in 2016 but has been continued under Courtauld Commitment 2025 (C2025) under the new title Your Business is Food: Don't Throw it Away (YBIF). Its objective is to reinvigorate interest and align the catering sector to the $\mathrm{C} 2025$ programme.

- The extension of the YBIF programme into the food processing and manufacturing sector from 2017 was formally launched nationally in 2018 following a series of consultations and development of guidance to associations for food manufacturers and to individual companies.

\section{Your business is food}

The initial Waste and Resources Action Programme (Wrap) Courtauld Commitment programmes have focused on the retail sector (Wrap, 2017). The hospitality and food service sector was engaged in 2012 through the HaFSA to undertake reductions in food waste and recycling of both packaging and food wastes. HaFSA has now been incorporated into C2025, the focus of which has now been more firmly placed on food waste prevention and reutilisation of surplus food.

The HaFSA initiative's objectives have now been subsumed into the $\mathrm{C} 2025$ programme to ensure its momentum continues with further developments, including most significantly YBIF (Wrap, 2018a). Measurement of food waste is an essential precursor for any business handling food in order to devise appropriate mechanisms to prevent food waste at the source, examine opportunities for surplus food redistribution and for segregating food waste for animal feed and anaerobic digestion.

There is now a YBIF $7 \mathrm{~d}$ tracking sheet which enables food businesses to look at their different sources of food waste over a typical week (Wrap, 2018b). However, following its promotion among potential restaurants and other businesses preparing food for a range of outlets Wrap introduced a $3 \mathrm{~d}$ version in order to meet the business concern that $7 \mathrm{~d}$ was too long to engage staff; it also provided a quick means for assessing their food waste to determine whether they needed to go for the full $7 \mathrm{~d}$ option. Almost all did utilise the new $3 \mathrm{~d}$ option and found that their food waste was costing them not only the collection and disposal costs but the costs of food purchase and preparation when they used the Wrap calculator to convert food waste generated to determine the overall financial cost.

The original YBIF was designed to prevent food waste generation in the hospitality and food service sector; it was developed during 2016 and launched in 2017 and has been adopted by a number of restaurant chains and individual businesses. Even the title of the initiative was recycled because in 2016, while the steering group was mulling over alternative titles for the new campaign, colleagues in South Australia had already developed a food waste prevention initiative entitled Your Business is Food and had prepared information material and graphics for the promotion of the campaign. They offered to allow Wrap to use the title and the promotional materials, which have been amended for British circumstances.

There are now a number of additional ways to simplify the process of calculating food waste from restaurants including 
Winnow and LeanPath, especially for plate waste, which is the most difficult element to assess in restaurants. From the work undertaken so far through a number of initiatives generated by C2025 and HaFSA partners and local projects such as the Greater London Authority's FoodSave scheme, there is a considerable body of research findings showing both the costs of food waste and the opportunities available to businesses to prevent and reutilise it. The accuracy and consistency of site-level food waste is an issue and most producers are reliant on the data provided by their waste management contractors. The continuing work of the hospitality and food service sector has continued through a focus group which has set up four task forces, including: data accuracy and development of measurement.

The measurement aspect was particularly important and this therefore required collaboration with waste management service contractors to examine how they were both collecting information from their clients and also providing information back to them about food waste weights collected. There were four of the leading waste management companies that were prepared to collaborate with the task force (TF) and goodquality data were provided through a questionnaire survey. As a result the guidelines regarding weighing of food waste could be updated and supplementary guidance about draft contractual terms between food waste producers and their potential waste management companies could be generated. Draft clauses for measurement, monitoring and reporting were also illustrated in the guidance. In addition, two useful case studies were provided: one by Suez and the other by Pizza Hut and their contractor Biffa.

TF2 focused on the measurement and the potential tools to assist the catering companies. From the feedback of HaFSA signatories and their members it was evident that companies were measuring their food waste, but many were suggesting that it would be good to have greater clarification on several matters regarding the definitions of food waste and measurement aspects. To ensure greater consistency Wrap is using the internationally agreed Food Loss and Waste Standard which was developed by the World Resources Institute (WRI, 2016), which Wrap also helped to establish. This standard was adopted by Tesco and has become a reporting requirement for Tesco's top 25 suppliers. It has also formed the basis for the Institute of Grocery Distribution's (IGD's) work, which has established a set of principles based on measurement from farm to fork.

Also there is a frequently asked questions section that people can use, for example, to make the distinction between food surplus and food waste - whereby the food surplus includes both redistribution and animal feed. It also includes how to classify catering food waste dropped on the floor during preparation and serving. However, there is a need for more case studies because, at the time of writing, the only case study is from the multinational Swedish retailer of homewares Ikea for their in-store restaurant facilities. This guidance is available on the Wrap website (Wrap, 2018c).

The third TF focused on the development of a self-assessment matrix which was due to be published in February 2018. It is an Excel file which allows the catering facilities to determine where they stand with regard to measuring food waste from no activity through good, better and best, with advice for getting from one stage to the next. This critically includes the important aspect of staff engagement (Wrap, 2018d).

\section{YBIF and the food processing and manufacturing sector}

In early 2018, the Wrap-sponsored initiative Your Business is Food was extended to the food manufacturing sector. In 2017, Wrap and a number of partner organisations, including the Food and Drink Federation, the IGD, Federation of Bakers and Dairy UK, decided to tackle the food waste that is generated further up the food supply chain in the food manufacturing sector through the C2025 YBIF Manufacturing Focus Group. Wrap has assessed that in order to reach the ambitious targets agreed under C2025, the main one being a reduction of $20 \%$ of environmental impact of food production, at least two-thirds of UK businesses will be required to take action. The extension of YBIF to the manufacturing sector is designed to help engage with the businesses that are members of $\mathrm{C} 2025$ partners and associations that are willing to join this initiative. An initial meeting of partners and supporters to agree a plan for future action was held in July 2017.

At this first meeting a modified version of the YBIF brochure, suitably adapted for the food manufacturing sector, was agreed in outline and also an action plan was undertaken to pre-test the guidance in the summer of 2017 and then fully pilot test the guidance with members of the $\mathrm{C} 2025$ partners during the autumn of 2017. At a further meeting in September 2017, the procedures for testing the guidance materials for the pilot sites discussed at the inaugural meeting were finalised. The pilots were to ensure that the materials were fit for purpose. These materials comprised

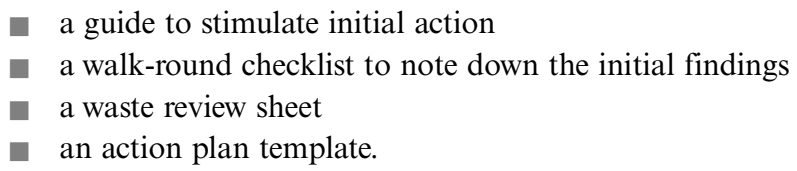

The IGD waste identification matrix is a series of Excel spreadsheets that allows the food processor to assess the financial costs of different sources of food waste in detail. 
At the beginning of September 2017, there were ten sites that had volunteered to be the initial guinea pigs, drawn from C2025 partners, including: the Food and Drink Federation, meat processors, bakers, two smaller fresh produce processors from the Co-op, a snacks manufacturer and a site volunteered by Premier Foods that needed to be brought up to standard as its other sites had already undertaken food waste audits. The sites for the pilots were mainly larger units.

Not surprisingly, it is difficult to engage with the smaller and medium-sized enterprises (SMEs) sector. Engaging the SMEs is where Scotland has an advantage over other UK administrations because by this time 60 SMEs in the food processing sector had already been audited, thanks to funding from the Scottish Government channelled through Zero Waste Scotland.

Following the results from these pilots, the guidance was modified as necessary so that the lessons learnt from the pilots could be assessed and any detailed changes to the starter pack could be made before it was disseminated through the C2025 partners in the autumn of 2017. Thereafter, a national Your Business is Food campaign aimed at the food processing sector was launched as one of the outputs of the C2025 programme at the annual C2025 meeting on 31 January 2018. This provided all food processors with access to the starter pack dealing with measuring and monitoring food waste. This would then enable all those varied producers and manufacturers of food products throughout the UK to access the promotional materials and the lessons of the pilot schemes that took place in the autumn of 2017.

\section{REFERENCES}

Wrap (Waste and Resources Action Programme) (2017) Courtauld Commitment 2025: Annual Review 2016/17. Wrap, Banbury, UK. See http://courtauldreview.wrap.org.uk/ (accessed 07/02/2018). Wrap (2018a) 'Your Business is Food: Don't Throw it Away' New Resources for Food Manufacturers. Wrap, Banbury, UK. See http://partners.wrap.org.uk/campaigns/your-business-is-food/ (accessed 07/02/2018).

Wrap (2018b) Food Waste Measurement Principles and Resources Guide. Wrap, Banbury, UK. See http://www.wrap.org.uk/content/ food-waste-measurement-principles-and-resources-guide (accessed 07/02/2018)

Wrap (2018c) http://www.wrap.org.uk/content/measuring-andreporting-food-waste-hospitality-and-food-service (accessed 28/02/2018).

Wrap (2018d) New Guidelines for Measuring and Reporting Food Waste in the Hospitality and Food Service Sector. Wrap, Banbury, UK. See http://www.wrap.org.uk/content/hospitality-and-food-serviceagreement-0 (accessed 07/02/2018).

WRI (World Resources Institute) (2016) Food Loss and Waste Accounting and Reporting Standard. WRI, Washington, DC, USA.

\section{How can you contribute?}

To discuss this paper, please email up to 500 words to the editor at journals@ice.org.uk. Your contribution will be forwarded to the author(s) for a reply and, if considered appropriate by the editorial board, it will be published as discussion in a future issue of the journal.

Proceedings journals rely entirely on contributions from the civil engineering profession (and allied disciplines). Information about how to submit your paper online is available at www.icevirtuallibrary.com/page/authors, where you will also find detailed author guidelines. 